\title{
Review Article \\ Methods of Cell Propulsion through the Local Stroma in Breast Cancer
}

\author{
Kerry J. Davies \\ Canniesburn Plastic Surgery Unit, Glasgow Royal Infirmary, Castle Street, Glasgow G4 OSF, UK \\ Correspondence should be addressed to Kerry J. Davies; kerry.angeletti@gmail.com
}

Received 6 January 2014; Accepted 18 March 2014; Published 7 April 2014

Academic Editor: Ian S. Fentiman

Copyright (C) 2014 Kerry J. Davies. This is an open access article distributed under the Creative Commons Attribution License, which permits unrestricted use, distribution, and reproduction in any medium, provided the original work is properly cited.

In the normal breast, cellular structures change cyclically in response to ovarian hormones. Cell proliferation, apoptosis, invasion, and differentiation are integral processes that are precisely regulated. Normal epithelial cells depend on the formation of intercellular adhesion contacts to form a continuous sheet of stratifying cell layers that are attached to one and other horizontally and vertically. Cells migrate by extending membrane protrusions to explore the extracellular space locating their targets in a chemotactic manner. The formation of cell protrusions is driven by the assembly of actin filaments at the leading edge. Reorganisation is regulated by a highly integrated signalling cascade that transduces extracellular stimuli to the actin filaments. This signalling cascade is governed by GTPases which act as molecular switches leading to actin polymerisation and the formation of filopodia and lamellipodia. This process is linked to downstream molecules known collectively as WASP proteins, which, in the presence of cortactin, form a complex leading to nucleation and formation of branched filaments. In breast cancer, the cortactin is over expressed leading to increased cellular motility and invasiveness. This hugely complex and integrated signalling cascade transduces extracellular stimuli. There are multiple genes related to cell motility which are dysregulated in human breast cancers.

\section{Introduction}

Breast cancer is by far the most frequent cancer among women with an estimated 1.38 million new cancer cases diagnosed in 2008 (23\% of all cancers) and ranks second overall (10.9\% of all cancers) [1, 2]. Breast cancer is estimated to be responsible for around 458,500 female deaths in 2008 or nearly one in seven (around 14\%) of all cancer deaths in women [3]. Cancer mortality is related to metastatic spread to other organs [4-6]. Therefore, early detection in order to improve breast cancer outcome and survival remains the cornerstone of breast cancer control [7].

The extracellular matrix (ECM) is composed of highly variable and dynamic components that regulate cell behavior. The protein composition and physical properties of the ECM govern cell fate through biochemical and biomechanical mechanisms. This requires carefully orchestrated and thorough regulation. In breast cancer, many ECM proteins are significantly deregulated and specific matrix components promote tumor progression and metastatic spread. Several ECM proteins that are associated with breast cancer development overlap substantially with a group of ECM proteins induced during the state of tissue remodeling such as mammary gland involution [8]. Understanding the regulatory role of the ECM will provide insight into mechanisms underlying normal and pathological development of the mammary gland [9].

Stromal tissue is composed of supporting cells and connective tissue, comprises a large component of the local microenvironment of many epithelial cell types, and influences several fundamental aspects of cell behaviour through both tissue interactions and niche regulation. The significance of the stroma in development and disease has been increasingly recognised [10].

The breast mesenchyme is comprised of complex connective tissue composed of heterogeneous cell types, including fibroblasts, adipocytes, immune cells, endothelial cells, pericytes, nerve cells, and acellular matrix components, such as collagen I, collagen III, collagen IV, proteoglycans, and glycoproteins [11]. The mammary gland is a dynamic tissue that undergoes significant changes throughout a woman's lifetime, especially during pregnancy and following the menopause [12]. The development of the breast is exquisitely sensitive to interactions between the epithelium and the stroma [13]. 
Stromal changes are required for the establishment of cancer [14]. The formation of vascular stroma in breast carcinoma is a process that involves complex reciprocal interactions among tumour cells, endothelial cells, and stromal cells [15].

Epithelial tumours lose their tissue organisation, become differentiated, and secrete abnormal quantities of ECM in a process that resembles wound healing and that is connected to the invasive and metastatic capacity of the primary tumour [16]. Underlying these events is a process known as epithelialmesenchymal-transition (EMT) which can be activated during chronic inflammation, for example, in chronic wounds or in cancer tissues. Key cellular alterations that occur during EMT include the loss of cell-cell adhesions and the change in the supporting cellular polarity. These changes affect the cells ability to support collective or individual cell migration [16].

In the EMT processes, epithelial cells gain mesenchymal properties and exhibit reduced intercellular adhesion and increased motility; they can also break through the basal membrane and migrate over long distances owing to profound changes in their cytoskeleton architecture [17]. This review focuses on how breast cancer cells undergo changes that enable them to become motile and break free of the basement membrane in a process that can lead ultimately to metastasis.

\section{Discussion}

2.1. The Metastatic Cell. Tumours can be defined by their uncontrolled and invasive growth but their phenotype is regulated in a complex fashion based on interactions of the malignant cells with the tumour stroma including the ECM, the vasculature, and the resident immune system [18]. Initially, a cell within a colony is instructed to disrupt cadherinbased intercellular junctions and acquire a fibroblastoid, motile phenotype, initiating detachment from the primary site. This is enhanced by proteases which digest the basal lamina components and facilitate cell movement through the ECM [19].

2.2. Cadherins. Cadherins are a family of adhesion molecules that function in cell recognition, tissue morphogenesis, and tumour suppression [20]. Normal epithelial cells depend on the formation of intercellular adhesion contacts to form a continuous sheet of stratifying cell layers that are attached to one another both horizontally and vertically. Cadherins forming these contacts are abundant in adherens and desmosomal junctions [21] which allow epithelial cells to move together as a sheet during wound repair and angiogenesis. This is known as collective migration and can be found in certain epithelial cell tumours. As epithelial cancer cells progress, the function of cell-cell junction protein is suppressed allowing individual cells to separate and migrate independently. This is known as individual cell migration, which can be subdivided into mesenchymal and amoeboid migration. In mesenchymal migration, the cell forms membrane protrusions at the leading edge and adheres to the substrate in an integrin-dependent manner. They release ECM degrading enzymes to remodel the ECM and form a path. Amoeboid migration occurs when the cell substrate adhesion is weak and is independent of integrin function. Cells move by actin-myosin contractile forces and move within the ECM by squeezing the cell body [22].

Cadherins have a large extracellular domain that binds to the same molecule on an adjacent cell. This results in dimerization of two cadherins on the same cell surface resulting in strong and stable adhesive forces [23]. E- and PCadherins are responsible for light cell-to-cell junctions in epithelia known as adherens junctions. N-Cadherin, found mainly in neural tissues and fibroblasts, is less stable and more dynamic [20]. E-Cadherin mediated cell-cell adhesions limit cell motility. The loss of E-Cadherin in epithelial cancers leads to disruption of tight junctions. This disrupts cell adhesion plaques enabling tumour cells to disengage from the primary mass and disseminate by individual cell migration [24]. In the mammary gland, E-Cadherin is cleaved by targeted expression of stromelysin-1: matrix metalloproteinase 9 (MMP 9). This is due to the interaction of N-Cadherin with the FGF receptor on the cell surface that increases transcription of MMP 9 leading to increased cellular invasiveness. In experimental models using mice over expression of E-Cadherin impairs invasiveness of tumour cells [20].

$\mathrm{N}$-Cadherin mediated cell-cell adhesion, in contrast to E-Cadherin, is required for collective cell migration [25]. Normally membrane extensions are suppressed in cells that are in contact with at least two neighbouring cells at the anterior and posterior ends [25]. This results in cell polarity. $\mathrm{N}$-Cadherin mediated cell-cell adhesion allows cells to move in sheets and functions in directional migration and contact inhibition. Upregulated N-Cadherin in breast cancer cells creates a state of dynamic adhesion that allows both attachment and detachment of individual cells from the primary tumour [26]. P-Cadherin is present in the highly mitotic terminal end ducts. P-Cadherin adhesion is important in mammary gland growth control and when disrupted can enhance cell invasion and tumour aggressiveness [27].

2.3. Matrix Metalloproteinases. The matrix metalloproteinases (MMPs) are a family of at least 28 zinc dependent endopeptidases. They degrade proteins in the ECM [28] leading to connective tissue dissolution. They are synthesised both by breast cancer cells and the surrounding stromal cells that may interact with each other [29], and it is likely that cancer cells are able to stimulate production by fibroblasts in a paracrine fashion [30]. In the normal breast, ECM remodelling is a prerequisite to ductal progression, and MMP activity facilitates this progression by removing or breeching the basement membrane and stromal matrix [31]. MMPs are implicated in cancer invasion and metastasis [32] and can be subdivided into four groups depending on their substrate specificity and domain organisations.

2.4. Rho Family Small GTPases. Once the ECM has been degraded, it paves the way for cells to move within its framework. Reorganisation of cortical actin filaments is regulated by a highly integrated signalling cascade that transduces extracellular stimuli to the actin filaments. This signalling 
cascade is governed by small GTPases [33]. Rho family GTPases regulate cytoskeletal dynamics by cycling between inactive GDP-bound and active GTP-bound states [34]. When cells are stimulated by growth factors, bound GDP is exchanged for GTP. In the GTP-bound state, Rho family small GTPases are active and interact with specific downstream effectors [22]. Rho GTPase members, including RhoA, Cdc42, and Rac, act as molecular switches on which signalling inputs converge and are transduced into a coordinated array of output pathways leading to actin polymerisation [35] and control of the formation of filopodia (Cdc42), lamellipodia (Rac), and stress fibres (Rho) [22].

P13K (phosphatidylinositol-3 kinase) is activated in response to chemoattractants [36]. This causes asymmetry in the internal signalling of the cell establishing cell polarity which defines the leading edge of the cell [22]. During cell movement, Rac and Cdc42 stimulate the formation of protrusion at the leading edges of the cells and Rho A induces retraction at the tail ends of the cells [33]. Activated Rac and Cdc42 induce reorganisation of the actin cytoskeleton at the leading edge. Localised actin polymerisation at the leading edge pushes the membrane forward generating the locomotive force in migrating cells [22]. RhoA regulates stress fibre formation and focal adhesion assembly that results in actomycin contractility and contraction at the rear of the cell which leads to translocation of the cell body [37].

2.5. Filopodia, Lamellipodia, and Invadopodia. Cells migrate forward by extending membrane protrusions [38]. In normal cells, these extensions are used to explore the extracellular space and find their way towards their targets in chemotactic location. Metastatic cells use this in cell migration [39]. There are two types of actin filaments. Branched actin filaments are normally associated with cortical areas and nonbranched actin filaments such as stress fibres that are frequently associated with focal adhesions, adherent junctions, and filopodia [40].

Filopodia are thin cylinders containing tight bundles of long actin filaments which protrude from the cell extending tens of microns from the main cortex. They are oriented in the direction of propulsion. Parallel actin bundles form the core structure packed tightly with noncontractile bundles cross-linked by F-actin binding proteins [41]. Lamellipodia are thin propulsive sheets that dominate the leading edges of fibroblasts and other motile cells. Lamellipodia have characteristic ruffle edges due to the leading edges lifting up from the substrate and moving backwards. Actin filaments in lamellipodia are oriented in a cross-weave pattern between two sets of filaments oriented approximately forty-five degrees to the direction of propulsion [42]. Podosomes are cell substrate adhesion sites which are usually observed in adhesive cells, for example, osteoclasts and macrophages. Podosomes are termed invadopodia in oncogene-transformed cells [22]. Invadopodia have the capacity to degrade the underlying matrix [43]. This distinction between podosomes and invadopodia is obscure. As invadopodia persist longer than podosomes, podosomes are thought to be the precursors of invadopodia [22].
2.6. WASPs. The formation of cell protrusions is driven by assembly of actin filaments at the leading edge [38]. During development, dynamic remodelling of the cytoskeleton allows the precise placement and orientation of developing tissues. There are many physiological signals that can trigger changes in cell motility and shape [44]. Although Rac and Cdc42 are known to be essential for cell movement, the downstream molecules involved in actin filament reorganisation are known as the WASP family of proteins [33]. WiskottAldrich syndrome is an X-chromosome linked hereditary disease that is characterised by thrombocytopenia, eczema, and immunodeficiency. The gene that was mutated was isolated and named Wiskott-Aldrich syndrome protein (WASP) [45]. To date, five mammalian WASP family proteins have been identified; WASP, neural WASP (N-WASP), and WAVE (WASP family verprolin-homologous proteins) 1, 2, and 3 [22]. These proteins have been identified as the link between the small GTPases and the actin cytoskeleton [33].

WASP binds to Cdc42 through its GBD/CRIB (GTPase binding domain/Cdc42 and Rac interactive binding domain) [33]. This brings about a conformational change in the WASP protein exposing the VCA (verprolin homology, cofilin homology, and acidic region) enabling G-actin and the Arp2/3 complex to promote actin nucleation and formation of protrusive structures such as filopodia and lamellipodia [36].

2.7. The Arp2/3 Complex. The Arp $2 / 3$ complex is a stable assembly of seven protein subunits. This complex binds to the sides of actin filaments and is concentrated at the leading edge of motile cells [46]. WASP, N-WASP, and WAVE share common biochemical features that enable them to form a tripartite unit with G-actin and the Arp2/3 complex [35]. The Arp $2 / 3$ complex multiplies actin filaments by branching when activated. It is responsible for the dendritic filament array observed in lamellipodia [39] and drives the leading edge of migrating cells. The Arp2/3 activating region of the WASP family protein binds with an active monomer resulting in rapid ATP hydrolysis on Arp2 and the nucleation of a new actin filament on the side of a preexisting filament [47]. Breast cancer with high histological grade has irregular morphology consisting of excessive protrusions due to the assemblages of branched actin filaments [48]. Aberrant expression of Arp2/3 in breast cancer leads to multiple branched actin filaments and leads to an increase in both local progression and poor disease outcome [49].

2.8. Cortactin. Cortactin is an actin associated scaffolding protein that regulates cell migration [50]. In the presence of both cortactin and WASP proteins, cortactin facilitates the release of activated WASPs by binding directly to the Arp $2 / 3$ complex [40] and activates it to promote nucleation of actin filaments [51] promoting and stabilising branched actin filaments [40]. In breast cancer cells, cortactin is associated with the invadopodium [51], and overexpression of cortactin leads to increased cellular motility and invasiveness [50]. Cortactin was found to be overexpressed in mammary tumours where 
its expression correlates with increased tumour invasiveness [50].

Research into WASPs and their interactions with other downstream effectors has shown them to be a hugely complex and highly integrated signalling cascade that transduces extracellular stimuli to the actin filaments. There are multiple genes involved in WASP related cell motility which are deregulated in human breast cancers.

2.9. Tumour Associated Macrophages (TAMs). It has been widely shown that breast tumours contain marked leukocytic infiltration that significantly correlates with poor prognosis. The majority of these cells are macrophages and CSF-1 (colony stimulating factor-1) is the main chemoattractant for these cells [52]. TAMs have been shown to promote the migratory phenotype of carcinoma cells, and macrophages are known to form podosomes and invadopodia [43]. In normal tissues, injury results in the local expression of a wide variety of growth factors one of which is CSF-1 [53].

CSF-1 was first identified as a haematopoietic growth factor that stimulates the proliferation, differentiation, and survival of monocytes, macrophages, and their bone progenitors [52]. In damaged tissues, CSF-1 attracts monocytes and stimulates them to differentiate into macrophages in order to mediate an immune response, kill pathogens, stimulate angiogenesis, and affect tissue repair [53]. In the lactating breast epithelium, very high levels of CSF-1 are expressed compared to undetectable levels found in resting breast epithelium [52] as CSF-1 produced by macrophages is implicated in the development of terminal end buds which grow out of the mammary breast ducts [53].

Sapi (2004) [52] reported that 58\% of all breast cancers and $85 \%$ of invasive breast carcinomas expressed high levels of CSF-1, and this expression was clearly localised in the neoplastic epithelial cells of the tumours as well as in the stromal macrophages. Pollard (2004) [53] suggests that there is a correlation between the expression of CSF-1 and poor prognosis in breast cancers which is due to the availability of CSF-1 to recruit and modulate the behaviour of TAMs. TAMs comigrate with carcinoma cells due to the presence of a CSF-1 EGF paracrine loop; carcinoma cells secrete CSF-1 and express the EGF receptor and macrophages secrete EGF and express the CSF-1 receptor stimulating each other to migrate via EGF activation of the N-WASP signalling pathway and formation of invadopodia [43].

2.10. Angiogenesis. Tumours require angiogenesis to grow beyond a certain size and TAMs have been shown to cluster in avascular areas correlating with high levels of angiogenesis and decreased relapse-free and overall survival rates in patients with breast carcinoma [53]. Hypoxia upregulates local cytokines and CSF-1, and these are chemotactic to the TAMs which migrate to the area where they stimulate angiogenesis by expressing VEGF and other growth factors and recruit haematopoietic cells such as mast cells and neutrophils [53].

High levels of macrophage infiltrate in breast tumours correlate with a poor prognosis, and hypoxic tumours have a higher invasive capacity and poorer prognosis than welloxygenated tumours [54]. Macrophages secrete MMP 9 and their podosomes are capable of directly degrading the pericellular ECM [43]. TAMs also promote tumour invasion by secreting uPA (urokinase plasminogen activator). uPA initiates a proteolytic cascade that results in the conversion of plasminogen to plasmin which mediates proteolysis [53]. uPA further recruits MMPs 9 and 2 enabling the remodelling of collagen type IV, the major constituent of basement membranes [54]. Breast tumour cells are then free to flow out of the ductally constrained tumour mass into the surrounding stroma and subsequently gaining access to the vasculature with the ability to colonise distant sites. High levels of uPA are associated with poor prognosis in breast cancer, and an elevated serum uPA is an established prognostic factor used for determining treatment-based decisions in early breast cancer [53].

\section{Conclusion}

As this review has highlighted, the movement of cells within the breast stroma is a hugely complex and a highly integrated process. There are multiple genes related to cell motility and these become dysregulated in breast cancers allowing cells to break down intercellular adhesive forces, cleave molecules in the ECM, and form motile invadopodia facilitating progression through the stroma. They are chemotactic and recruit other cells to stimulate angiogenesis, growth factors, and proteolysis enabling the breast cancer cells to break free of the basement membrane, gain access to the vasculature, and colonise distant sites.

\section{Conflict of Interests}

The authors declare that there is no conflict of interests.

\section{References}

[1] http://globocan.iarc.fr/factsheets/cancers/breast.asp.

[2] http://www.wcrf.org/cancer_statistics/world_cancer_statistics. php.

[3] http://www.cancerresearchuk.org/cancer-info/cancerstats/ types/breast/mortality/\#trends.

[4] E. J. Kim and D. M. Helfman, "Characterization of the metastasis-associated protein, S100A4: roles of calcium binding and dimerization in cellular localization and interaction with myosin," Journal of Biological Chemistry, vol. 278, no. 32, pp. 30063-30073, 2003.

[5] K. Pantel and R. H. Brakenhoff, "Dissecting the metastatic cascade," Nature Reviews Cancer, vol. 4, no. 6, pp. 448-456, 2004.

[6] S. M. Ali, H. A. Harvey, and A. Lipton, "Medical management of bone metastasis. Metastatic breast cancer. Overview of treatment," Clinical Orthopaedics and Related Research, vol. 515S, no. 415, pp. S132-S137, 2003.

[7] http://www.who.int/cancer/detection/breastcancer/en/index .html. 
[8] T. Oskarsson, "Extracellular matrix components in breast cancer progression and metastasis," Breast, vol. 22, no. 2, pp. S66S72, 2013.

[9] Q. K. Chen, K. Lee, D. C. Radisky, and C. M. Nelson, "Extracellular matrix proteins regulate epithelial-mesenchymal transition in mammary epithelial cells," Differentiation, vol. 86, no. 3, pp. 126-132, 2013.

[10] B. A. Howard and P. Lu, "Stromal regulation of embryonic and postnatal mammary epithelial development and differentiation," Seminars in Cell \& Developmental Biology, 2014.

[11] G. B. Silberstein, "Tumour-stromal interactions. Role of the stroma in mammary development," Breast Cancer Research, vol. 3, no. 4, pp. 218-223, 2001.

[12] J. McCready, L. M. Arendt, J. A. Rudnick, and C. Kuperwasser, "The contribution of dynamic stromal remodeling during mammary development to breast carcinogenesis," Breast Cancer Research, vol. 12, no. 3, article 205, 2010.

[13] A. Howell, G. Landberg, and J. Bergh, "Breast tumour stroma is a prognostic indicator and target for therapy," Breast Cancer Research, vol. 11, supplement 3, article S16, 2009.

[14] M. H. Barcellos-Hoff and D. Medina, "New highlights on stroma-epithelial interactions in breast cancer," Breast Cancer Research, vol. 7, no. 1, pp. 33-36, 2005.

[15] L. F. Brown, A. J. Guidi, S. J. Schnitt et al., "Vascular stroma formation in carcinoma in situ, invasive carcinoma, and metastatic carcinoma of the breast," Clinical Cancer Research, vol. 5, no. 5, pp. 1041-1056, 1999.

[16] A. Moustakas and P. Heldin, "TGF $\beta$ and matrix-regulated epithelial to mesenchymal transition," Biochimica et Biophysica Acta, 2014.

[17] Y. Wang and B. P. Zhou, "Epithelial-mesenchymal transition-a hallmark of breast cancer metastasis," Cancer Hallmarks, vol. 1, no. 1, pp. 38-49, 2013.

[18] C. H. Stuelten, S. DaCosta Byfield, P. R. Arany, T. S. Karpova, W. G. Stetler-Stevenson, and A. B. Roberts, "Breast cancer cells induce stromal fibroblasts to express MMP-9 via secretion of TNF- $\alpha$ and TGF- $\beta$," Journal of Cell Science, vol. 118, no. 10, pp. 2143-2153, 2005.

[19] P. M. Comoglio and L. Trusolino, "Invasive growth: from development to metastasis," Journal of Clinical Investigation, vol. 109, no. 7, pp. 857-862, 2002.

[20] R. B. Hazan, R. Qiao, R. Keren, I. Badano, and K. Suyama, "Cadherin switch in tumor progression," Annals of the New York Academy of Sciences, vol. 1014, pp. 155-163, 2004.

[21] B. L. Ziober, S. S. Silverman Jr., and R. H. Kramer, "Adhesive mechanisms regulating invasion and metastasis in oral cancer," Critical Reviews in Oral Biology and Medicine, vol. 12, no. 6, pp. 499-510, 2001.

[22] D. Yamazaki, S. Kurisu, and T. Takenawa, "Regulation of cancer cell motility through actin reorganization," Cancer Science, vol. 96, no. 7, pp. 379-386, 2005.

[23] M. Overduin, T. S. Harvey, S. Bagby et al., "Solution structure of the epithelial cadherin domain responsible for selective cell adhesion," Science, vol. 267, no. 5196, pp. 386-389, 1995.

[24] Y. L. Chao, C. R. Shepard, and A. Wells, "Breast carcinoma cells re-express E-cadherin during mesenchymal to epithelial reverting transition," Molecular Cancer, vol. 9, article 179, 2010.

[25] W. Shih and S. Yamada, "N-Cadherin mediated cell-cell adhesion promotes cell migration in a three-dimensional matrix," Journal of Cell Science, vol. 125, pp. 3661-3670, 2012.
[26] R. B. Hazan, G. R. Phillips, R. F. Qiao, L. Norton, and S. A. Aaronson, "Exogenous expression of $\mathrm{N}$-cadherin in breast cancer cells induces cell migration, invasion, and metastasis," Journal of Cell Biology, vol. 148, no. 4, pp. 779-790, 2000.

[27] J. Paredes, A. L. Correia, A. S. Ribeiro, A. Albergaria, F. Milanezi, and F. C. Schmitt, "P-cadherin expression in breast cancer: a review," Breast Cancer Research, vol. 9, no. 5, article 214, 2007.

[28] M. J. Duffy, T. M. Maguire, A. Hill, E. McDermott, and N. O'Higgins, "Metalloproteinases: role in breast carcinogenesis, invasion and metastasis," Breast Cancer Research, vol. 2, no. 4, pp. 252-257, 2000.

[29] A. Lebeau, A. G. Nerlich, U. Sauer, R. Lichtinghagen, and U. Löhrs, "Tissue distribution of major matrix metalloproteinases and their transcripts in human breast carcinomas," Anticancer Research, vol. 19, no. 5B, pp. 4257-4264, 1999.

[30] N. Reimers, K. Zafrakas, V. Assmann et al., "Expression of extracellular matrix metalloproteases inducer on micrometastatic and primary mammary carcinoma cells," Clinical Cancer Research, vol. 10, no. 10, pp. 3422-3428, 2004.

[31] J. E. Fata, A. T.-V. Ho, K. J. Leco, R. A. Moorehead, and R. Khokha, "Cellular turnover and extracellular matrix remodeling in female reproductive tissues: functions of metalloproteinases and their inhibitors," Cellular and Molecular Life Sciences, vol. 57, no. 1, pp. 77-95, 2000.

[32] T. Shiomi and Y. Okada, "MT1-MMP and MMP-7 in invasion and metastasis of human cancers," Cancer and Metastasis Reviews, vol. 22, no. 2-3, pp. 145-152, 2003.

[33] T. Takenawa and H. Miki, "WASP and WAVE family proteins: key molecules for rapid rearrangement of cortical actin filaments and cell movement," Journal of Cell Science, vol. 114, no. 10, pp. 1801-1809, 2001.

[34] M. Parsons, J. Monypenny, S. M. Ameer-Beg et al., "Spatially distinct binding of Cdc42 to PAK1 and N-WASP in breast carcinoma cells," Molecular and Cellular Biology, vol. 25, no. 5, pp. 1680-1695, 2005.

[35] T. E. B. Stradal, K. Rottner, A. Disanza, S. Confalonieri, M. Innocenti, and G. Scita, "Regulation of actin dynamics by WASP and WAVE family proteins," Trends in Cell Biology, vol. 14, no. 6, pp. 303-311, 2004.

[36] J. Sturge, J. Hamelin, and G. E. Jones, "N-WASP activation by a $\beta$-integrin-dependent mechanism supports $\mathrm{P} 13 \mathrm{~K}$-independent chemotaxis stimulated by urokinase-type plasminogen activator," Journal of Cell Science, vol. 115, no. 4, pp. 699-711, 2002.

[37] S. Kurisu, S. Suetsugu, D. Yamazaki, H. Yamaguchi, and T. Takenawa, "Rac-WAVE2 signaling is involved in the invasive and metastatic phenotypes of murine melanoma cells," Oncogene, vol. 24, no. 8, pp. 1309-1319, 2005.

[38] M. Lorenz, H. Yamaguchi, Y. Wang, R. H. Singer, and J. Condeelis, "Imaging sites of N-WASP activity in lamellipodia and invadopodia of carcinoma cells," Current Biology, vol. 14, no. 8, pp. 697-703, 2004.

[39] M.-F. Carlier, S. Wiesner, C. Le Clainche, and D. Pantaloni, "Actin-based motility as a self-organized system: mechanism and reconstitution in vitro," Comptes Rendus-Biologies, vol. 326, no. 2, pp. 161-170, 2003.

[40] J. Lin, J. Liu, Y. Wang et al., "Differential regulation of cortactin and N-WASP-mediated actin polymerization by missing in metastasis (MIM) protein," Oncogene, vol. 24, no. 12, pp. 20592066, 2005.

[41] A. Yamagishi, M. Masuda, T. Ohki, H. Onishi, and N. Mochizuki, "A novel actin bundling/filopodium-forming 
domain conserved in insulin receptor tyrosine kinase substrate p53 and missing in metastasis protein," Journal of Biological Chemistry, vol. 279, no. 15, pp. 14929-14936, 2004.

[42] T. J. Mitchison and L. P. Cramer, "Actin-based cell motility and cell locomotion,” Cell, vol. 84, no. 3, pp. 371-379, 1996.

[43] H. Yamaguchi, F. Pixley, and J. Condeelis, "Invadopodia and podosomes in tumor invasion," European Journal of Cell Biology, vol. 85, no. 3-4, pp. 213-218, 2006.

[44] J. A. Woodings, S. J. Sharp, and L. M. Machesky, "MIM-B, a putative metastasis suppressor protein, binds to actin and to protein tyrosine phosphatase $\delta$," Biochemical Journal, vol. 371, no. 2, pp. 463-471, 2003.

[45] H. Miki and T. Takenawa, "Regulation of actin dynamics by WASP family proteins," Journal of Biochemistry, vol. 134, no. 3, pp. 309-313, 2003.

[46] R. D. Mullins, J. A. Heuser, and T. D. Pollard, "The interaction of Arp2/3 complex with actin: nucleation, high affinity pointed end capping, and formation of branching networks of filaments," Proceedings of the National Academy of Sciences of the United States of America, vol. 95, no. 11, pp. 6181-6186, 1998.

[47] M. J. Dayel and R. D. Mullins, "Activation of Arp2/3 complex: addition of the first subunit of the new filament by a WASP protein triggers rapid ATP hydrolysis on Arp2," PLoS Biology, vol. 2, no. 4, pp. 476-485, 2004.

[48] K. Iwaya, K. Norio, and K. Mukai, "Coexpression of Arp2 and WAVE2 predicts poor outcome in invasive breast carcinoma," Modern Pathology, vol. 20, no. 3, pp. 339-343, 2007.

[49] M. Moazzam, L. Ye, H. Kynaston, R. Mansel, and W. Jiang, "Aberrant expression of Arp2/3 in breast cancer and the association with disease progress," Cancer Research, vol. 69, no. 24, p. $6169,2009$.

[50] J. R. Kowalski, C. Egile, S. Gil, S. B. Snapper, R. Li, and S. M. Thomas, "Cortactin regulates cell migration through activation of N-WASP," Journal of Cell Science, vol. 118, no. 1, pp. 79-87, 2005.

[51] T. Uruno, J. Liu, P. Zhang et al., "Activation of Arp2/3 complexmediated actin polymerization by cortactin," Nature Cell Biology, vol. 3, no. 3, pp. 259-266, 2001.

[52] E. Sapi, "The Role of CSF-1 in normal physiology of mammary gland and breast cancer: an update," Experimental Biology and Medicine, vol. 229, no. 1, pp. 1-11, 2004.

[53] J. W. Pollard, "Tumour-educated macrophages promote tumour progression and metastasis," Nature Reviews Cancer, vol. 4, no. 1, pp. 71-78, 2004.

[54] M. J. Grimshaw, T. Hagemann, A. Ayhan, C. E. Gillett, C. Binder, and F. R. Balkwill, "A role for endothelin-2 and its receptors in breast tumor cell invasion," Cancer Research, vol. 64, no. 7, pp. 2461-2468, 2004. 


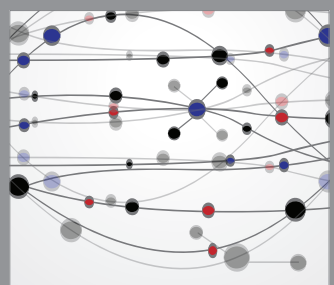

The Scientific World Journal
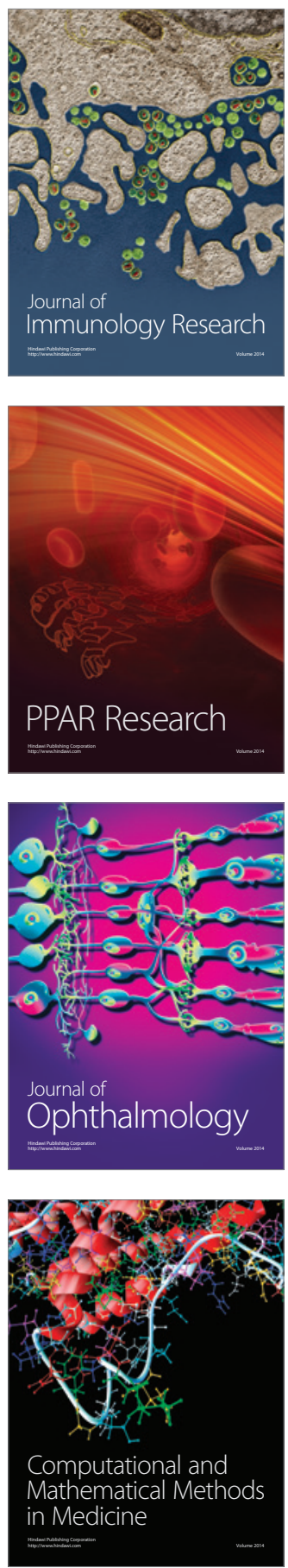

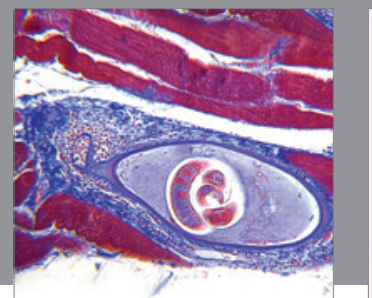

Gastroenterology

Research and Practice
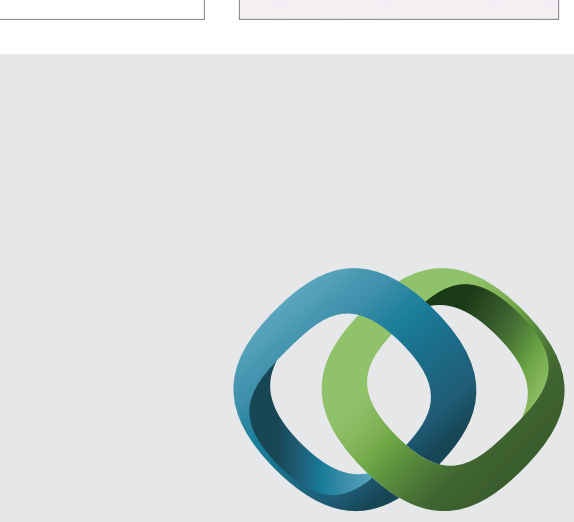

\section{Hindawi}

Submit your manuscripts at

http://www.hindawi.com
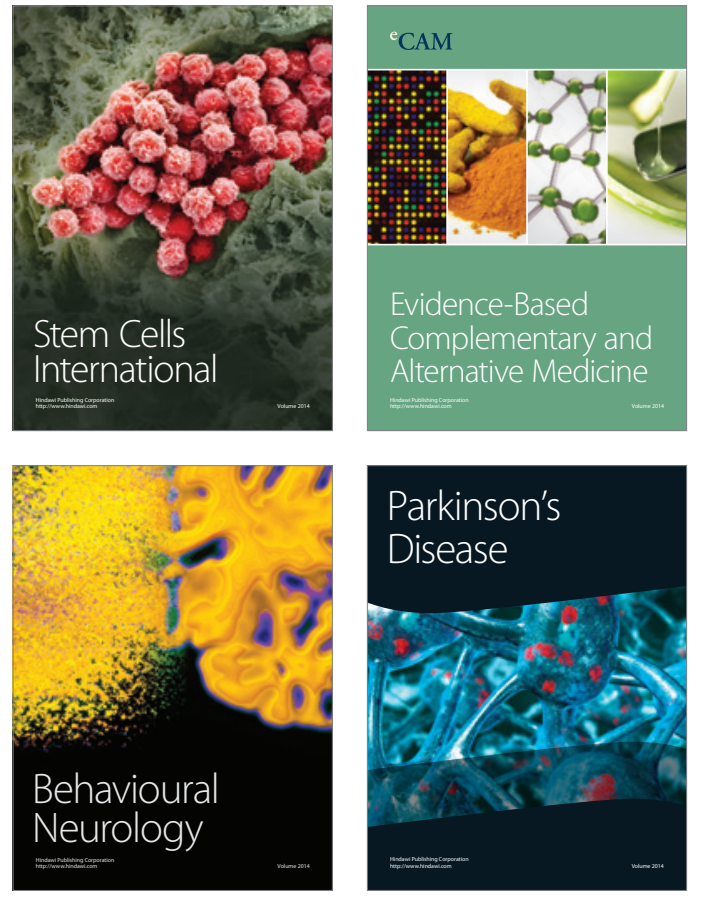
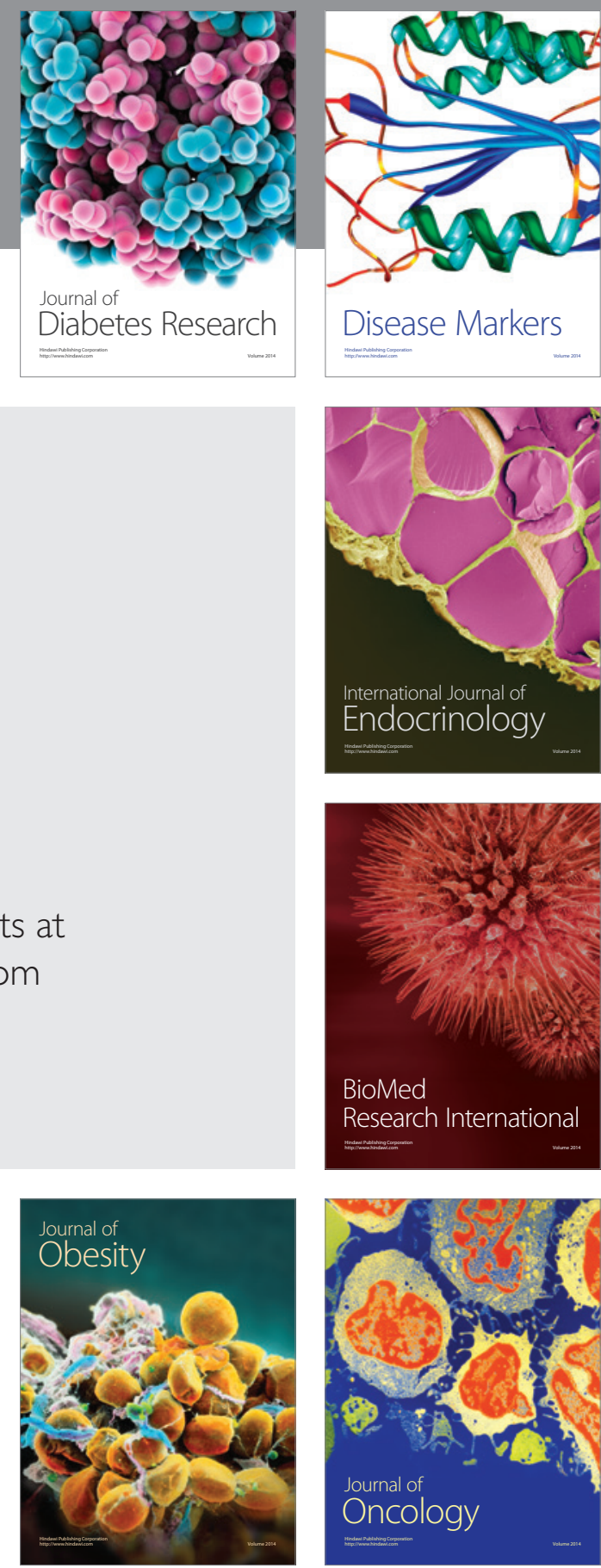

Disease Markers
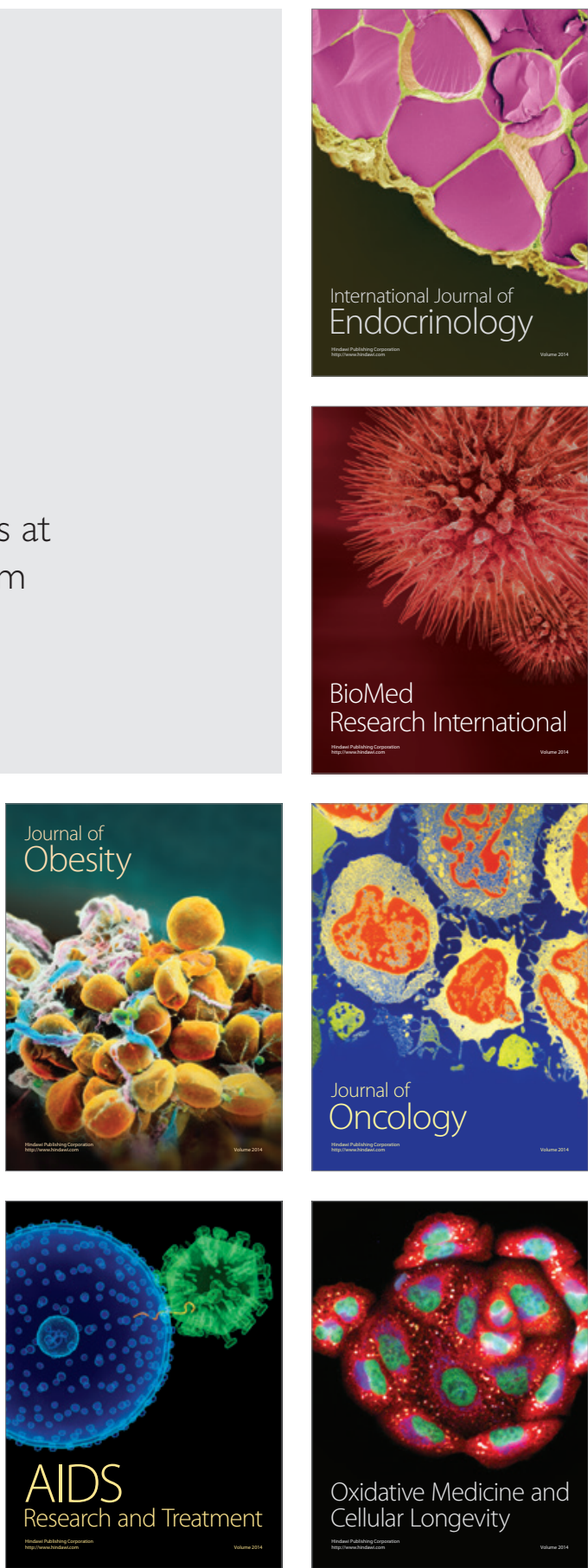